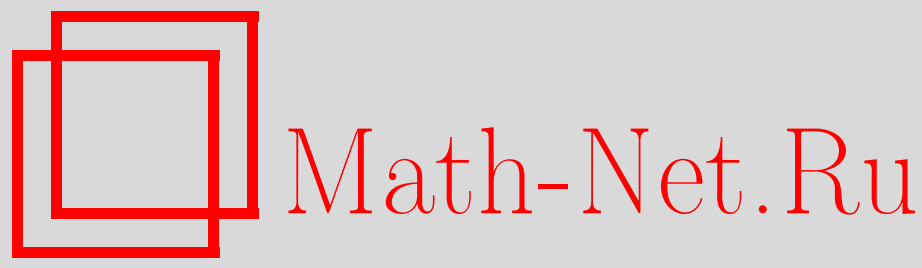

А. В. Юров, А. В. Асташенок, Метод линеаризации и новые классы точных решений в космологии, ТМФ, 2009, том 158, номер 2, 312-320

DOI: https://doi.org/10.4213/tmf6316

Использование Общероссийского математического портала Math-Net.Ru подразумевает, что вы прочитали и согласны с пользовательским соглашением http://www . mathnet.ru/rus/agreement

Параметры загрузки:

IP : 54.172 .240 .79

26 апреля 2023 г., 02:36:28

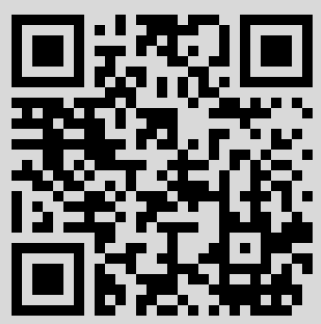




\title{
МЕТОД ЛИНЕАРИЗАЦИИ И НОВЫЕ КЛАССЫ ТОЧНЫХ РЕШЕНИЙ В КОСМОЛОГИИ
}

\begin{abstract}
Развит метод построения точных космологических решений уравнений Эйнштейна, основанный на их представлении в форме линейного дифференциального уравнения второго порядка. Метод позволяет, в частности, использовать произвольное известное решение для построения более общего решения, параметризованного набором из $3 N$ констант, где $N$ - произвольное натуральное число. Наличие большого числа свободных параметров может оказаться полезным для построения теоретической модели, приводящей к удовлетворительному согласию с результатами астрономических тестов. Аналогичными свойствами обладают космологические решения на бране Рэндалл-Сундрум I. Показано, что уже трехпараметрические решения в общем случае демонстрируют наличие инфляционных режимов, причем в отличие от изученных ранее двухпараметрических решений трехпараметрические решения могут описывать выход из инфляции без точной настройки параметров, а также наличие нескольких последовательных инфляционных фаз.
\end{abstract}

Ключевые слова: космология, инфляция, точные решения.

\section{1. ВВЕДЕНИЕ}

В работе [1] приводится метод построения и анализа точных космологических решений уравнений Эйнштейна, основанный на представлении их в форме линейного уравнения второго порядка (в дальнейшем мы будем ссылаться на эту процедуру как на метод линеаризации $)^{1)}$. Действительно, легко убедиться, что в случае плоской метрики Фридмана третья степень масштабного фактора $\psi=a^{3}$ будет удовлетворять уравнению

$$
\frac{d^{2} \psi}{d t^{2}}=\frac{9}{2}(\rho-p) \psi
$$

где $\rho$ - плотность, а $p$ - давление вещества, наполняющего вселенную. Здесь и далее мы пользуемся системой единиц, в которой $8 \pi G / 3=c=1$. В случае преобладания минимально связанного скалярного поля $\phi$ с потенциалом самодействия $V(\phi)$ и наличия космологической постоянной с плотностью $\Lambda$ уравнение (1) принимает вид,

1) Другие методы построения точных решений в космологии можно найти в работах [2]-[7].

${ }^{*}$ Российский государственный университет им. И. Канта, Калининград, Россия. E-mail: artyom_yurov@mail.ru, artyom.art@gmail.com 
формально совпадающий с уравнением Шредингера

$$
\frac{d^{2} \psi}{d t^{2}}=(U-\lambda) \psi
$$

где потенциал $U(t)=9 V$, а спектральный параметр $\lambda=-9 \Lambda$. В (2) подразумевается, что величина $V$ зависит от времени: $V(t)=V(\phi(t))$, эту функцию $V(t)$ авторы работы [1] называют историей потенциала. Задавая явный вид $U(t)$ вместе с соответствующими начальными условиями, можно найти общее решение уравнения (2). Важным следствием этого исследования является указание на независимость или слабую зависимость режима от типа потенциала, что весьма существенно для всей теории. K сожалению, проблема выхода из инфляции оказалась значительно сложнее и, по-видимому, требует в рамках описываемого подхода дополнительных предположений ${ }^{2)}$. Исследование уравнения (2) в приложении к космологии было продолжено в работах [8], [9], где для построения новых точных решений использовалось преобразование Дарбу ${ }^{3)}$.

В настоящей работе мы предлагаем модификацию данного метода. Из уравнений Эйнштейна следует, что для функции $\psi_{n}=a^{n}$, где $n-$ произвольное (необязательно целое) число, можно получить уравнение Шредингера с функцией $U_{n}$, которая является линейной комбинацией плотности и давления. Если полагать, что вселенная заполнена минимально связанным скалярным полем, то $U_{n}$ будет линейной комбинацией потенциала $V(\phi)$ и кинетического члена, и потому уже нецелесообразно называть $U_{n}$ историей потенциала. В дальнейшем будем ссылаться на величину $U_{n}$, как на "потенциал" (в кавычках), чтобы не путать его с потенциалом самодействия $V$.

Задавая "потенциал", можно получать решения для функции $\psi_{n}$ и таким образом находить масштабный фактор $a_{n}=\psi_{n}^{1 / n}$ как функцию времени. В общем случае решение уравнения Шредингера имеет вид

$$
\psi_{n}(t)=c_{1} \psi_{1}(t)+c_{2} \psi_{2}(t),
$$

т.е. зависит от двух произвольных констант. В свою очередь новый масштабный фактор зависит от трех параметров: $a=a\left(n, c_{1}, c_{2} ; t\right)$. Это обстоятельство можно использовать для построения многопараметрических решений уравнений Эйнштейна следующим образом: предположим, что величина $a(t)$ определена, скажем, на основании астрономических наблюдений. Возводя эту функцию в степень $n_{1}$, мы находим функцию, которая в (3) обозначена $\psi_{1}$. На следующем шаге мы вычисляем $\psi_{2}$, исходя из условия линейной независимости, записанного в виде уравнения

$$
\psi_{1} \frac{d \psi_{2}}{d t}-\psi_{2} \frac{d \psi_{2}}{d t}=1
$$

после чего находим трехпараметрическое решение $a=a\left(n_{1}, c_{1}^{(1)}, c_{2}^{(1)} ; t\right)$. Теперь можно повторить эту процедуру и вычислить шестипараметрическое решение

$$
a=a\left(n_{1}, c_{1}^{(1)}, c_{2}^{(1)} ; n_{2}, c_{1}^{(2)}, c_{2}^{(2)} ; t\right)
$$

2) Авторы цитируемой работы [1] предложили так модифицировать потенциалы, чтобы параметры оказались зависящими от температуры. Тогда выход на фридмановский режим можно описывать как фазовый переход в материи ранней вселенной.

3) В работе [10] аналогичная техника использовалась для построения точных решений на бране и в объемлющем пространстве, снабженном структурой орбиобразия. 
и т.д. После $N$ шагов мы находим решение, содержащее $3 N$ параметров. С другой стороны, в космологии Фридмана знание масштабного фактора как функции времени позволяет полностью вычислить все остальные характеристики, например параметр Хаббла, ускорение, плотность, давление и т.д. Важнейшая для тестирования моделей величина фотометрического расстояния является функцией шести параметров, поэтому, используя вышеописанную процедуру необходимое число раз, можно добиться согласования модели с наблюдениями.

$\mathrm{K}$ сожалению, эффективность данного подхода значительно снижается при рассмотрении фридмановских моделей с ненулевой пространственной кривизной. В этом случае при произвольных $n$ функция $\psi_{n}=a^{n}$ удовлетворяет не уравнению Шредингера, а уравнению с дополнительным нелинейным слагаемым. Для такого уравнения отсутствуют содержательные методы интегрирования, и потому затруднительно в общем случае найти решение, параметризованное двумя произвольными константами, что необходимо для полного исследования задачи, аналогичного проведенному в работе [1]. Было бы неплохо показать, что выводы работы [1] справедливы и при $k= \pm 1$. K счастью, для этого нет необходимости решать сложное нелинейное уравнение, о котором речь шла выше: достаточно выбрать $n=1$, а не $n=3$. В этом случае роль волновой функции играет сам масштабный фактор. Дело в том, что одно из двух космологических уравнений Эйнштейна в метрике Фридмана является линейным уравнением второго порядка с потенциалом, пропорциональным (со знаком минус) величине $\rho+3 p / c^{2}$. Более прозрачным является и физический смысл "потенциала": если космологический член выбрать равным нулю, то отрицательность потенциала означает выполнение сильного энергетического условия, а положительность - нарушение, приводящее, вообще говоря, к инфляции. Поэтому задача исследования инфляционных режимов становится гораздо более простой, даже при сравнении с плоскими $(k=0)$ моделями.

Работа организована следующим образом. В разделе 2 мы приводим точную формулировку процедуры линеаризации, т.е. сведе́ния уравнений Фридмана к уравнению Шредингера для произвольного $n$. Кроме того, мы доказываем аналогичное утверждение для космологии на бране Рэндалл-Сундрум I (RS-I). B разделе 3 мы приводим несколько примеров точных решений. Мы увидим, что трехпараметрические семейства решений оказываются значительно богаче по своим свойствам, чем исследованные в работе [1] двухпараметрические решения. В частности, такие "потенциалы" могут приводить к решениям, описывающим несколько инфляционных стадий. Возможно, наша Вселенная переживает одну из них.

\section{2. ПРОЦЕДУРА ЛИНЕАРИЗАЦИИ}

Рассмотрим уравнения Эйнштейна в метрике Фридмана:

$$
\begin{aligned}
\frac{\dot{a}^{2}}{a^{2}} & =\rho-\frac{k}{a^{2}}, \\
\frac{\ddot{a}}{a} & =-\frac{1}{2}(\rho+3 p) .
\end{aligned}
$$


Предположим, что $p=p(t), \rho=\rho(t) ; a=a(t)$ - решение этих уравнений при $k=0$. Тогда функция $\psi_{n}=a^{n}$ является решением уравнения Шредингера

$$
\ddot{\psi}_{n}=U_{n}(t) \psi_{n}
$$

где "потенциал"

$$
U_{n}(t)=n^{2} \rho-\frac{3 n}{2}(\rho+p)
$$

Если вселенная заполнена скалярным полем $\phi$ с лагранжианом $L=\dot{\phi}^{2} / 2-V(\phi)$, то

$$
U_{n}=\frac{n(n-3)}{2} \dot{\phi}^{2}+n^{2} V(\phi)
$$

ЗАМЕчАниЕ 1. Для $n=3$ "потенциал" $U_{3}=9 V(\phi(t))$. Этот случай был подробно исследован в работе [1], где, как мы уже отмечали, эта величина была названа историей потенциала, потому что $U_{3}$ появляется в уравнениях как функция времени $(U=U(t))$, а не как функция полевой переменной $\phi$. Тем не менее потенциал $U$ пропорционален $V$, поэтому создается впечатление, что физический смысл потенциала $U$ ясен. Если же $n \neq 3$, то $U_{n}$ оказывается некоторой линейной комбинацией кинетического члена $\dot{\phi}^{2} / 2$ и потенциала самодействия $V$ (см. (7), (8)), и физический смысл такого $U$ уже не вполне ясен.

Однако эффективность метода, предложенного в работе [1] (и развитого в работах [8], [9]), состоит в том и только в том, что сложная нелинейная задача сведена к линейному уравнению. Это позволяет найти полные двухпараметрические решения, которые демонстрируют наличие инфляции при чрезвычайно общих предположениях. Тот факт, что $U$ по сути совпадает с $V$, в цитируемых работах буквально нигде не используется, потому что это не играет никакой роли. Аналогично, в настоящей работе мы рассматриваем обобщение данного метода для случая произвольного $n$.

$\mathrm{K}$ тому же физический смысл "потенциала" $U_{3}$ представляется ясным только для вселенной, заполненной скалярным полем. Если рассмотреть вселенную, в которой доминирует, скажем, электромагнитное излучение, то смысл величины $U_{3}$ станет неопределенным.

ЗАмЕчАниЕ 2. Если допустить, что наряду с полями материи во вселенной присутствует ненулевая энергия вакуума с плотностью $\rho_{\Lambda} c^{2}$, то уравнение $(6)$ примет вид спектральной задачи

$$
\ddot{\psi}_{n}=\left(U_{n}(t)-\lambda_{n}\right) \psi_{n}
$$

где спектральный параметр $\lambda_{n}=-3 n^{2} \rho_{\Lambda} / 3$. Как и уравнение $(2)$, уравнение (9) допускает постановку задачи на собственные значения и собственные функции, если дополнить это уравнение однородными начальными условиями. Как отмечено в работе [1], уравнение (2) по форме совпадает с задачами квантовой механики для дискретного спектра. Тот факт, что для каждой такой задачи возникает лишь ограниченный или счетный набор допустимых значений космологической постоянной (если дополнить уравнение однородными начальными условиями), может внести ясность в вопрос о реальном значении космологической постоянной. 
Заметим, что если известно решение (9), то, используя (4), (9), можно найти скалярное поле и потенциал:

$$
\begin{aligned}
\phi(t) & = \pm \frac{\sqrt{2}}{\sqrt{3 n}} \int d t \sqrt{\frac{\dot{\psi}_{n}^{2}}{\psi_{n}^{2}}-U_{n}+\lambda_{n}}, \\
V(t) & =\frac{1}{3}\left(\frac{U_{n}}{n}+\frac{3-n}{n^{2}}\left(\frac{\dot{\psi}_{n}^{2}}{\psi_{n}^{2}}+\lambda_{n}\right)\right) .
\end{aligned}
$$

Из этих выражений можно получить зависимость $V=V(\phi)$, хотя понятно, что далеко не всегда это можно сделать в явном виде. В общем случае решение уравнения (6) имеет форму

$$
\Psi_{n}=c_{1} \psi_{n}+c_{2} \hat{\psi}_{n}
$$

где $\hat{\psi}_{n}$ есть линейно независимое решение с тем же потенциалом:

$$
\hat{\psi}_{n}(t)=\psi_{n}(t) \int^{t} \frac{d t^{\prime}}{\psi_{n}^{2}\left(t^{\prime}\right)} \equiv \psi_{n}(t) \xi(t) .
$$

Уравнение (12) позволяет доказать следующее

УтВЕРЖДЕНИЕ. Пусть $a=a(t)$ - решение уравнений (4), (5) при $k=0$ и соответствующих $\rho$ и . Тогда трехпараметрическая функиия $a_{n}=a\left(t ; c_{1}, c_{2}, n\right)$ вида

$$
a_{n}=a\left(c_{1}+c_{2} \int \frac{d t}{a^{2 n}}\right)^{1 / n}
$$

будет решением уравнений (4), (5) с новой плотностью энергии $\rho_{n}$ и давлением $p_{n}$, которые удовлетворяют следующему условию:

$$
n^{2} \rho_{n}-\frac{3 n}{2}\left(\rho_{n}+\frac{p_{n}}{c^{2}}\right)=n^{2} \rho-\frac{3 n}{2}\left(\rho+\frac{p}{c^{2}}\right) .
$$

ЗАмЕчАНИЕ 3. В общем случае это утверждение справедливо для случая $k=0$. Если $k= \pm 1$, то утверждение будет выполняться, только если $n=0,1$.

ЗАмечАниЕ 4. Аналогичное утверждение можно сформулировать для решений, описывающих брану RS-I. В этом случае система уравнений Фридмана модифицируется за счет учета натяжения на бране $\sigma$ и принимает вид

$$
\begin{aligned}
\left(\frac{\dot{a}}{a}\right)^{2} & =\rho\left(1+\frac{\rho}{2 \sigma}\right), \\
-2 \frac{\ddot{a}}{a} & =\rho+3 p+\frac{\rho}{\sigma}(2 \rho+3 p) .
\end{aligned}
$$

Легко убедиться, что функция $\psi_{n} \equiv a^{n}$ удовлетворяет линейному уравнению Шредингера

$$
\frac{\ddot{\psi}_{n}}{\psi_{n}}=W_{n}
$$


с потенциалом

$$
\begin{aligned}
W_{n} & =\frac{n}{2}\left(2 n \rho-3(\rho+p)+\frac{\rho}{\lambda}(n \rho-3(\rho+p))\right)= \\
& =\frac{n}{2}\left(2 n(K+V)-6 K+\frac{1}{\lambda}(K+V)(n(K+V)-6 K)\right),
\end{aligned}
$$

где $V=V(\phi), K=\dot{\phi}^{2} / 2$. Отсюда следует, что описанный выше метод генерации $3 N$-параметрических решений может быть применен и для космологии на бране. Пример точного двухпараметрического решения (с $n=3)$ приведен в работе [11]. В этой же работе описана процедура линеаризации для простейшей анизотропной космологической модели.

Ниже, следуя работе [1], мы рассмотрим несколько примеров точных решений, основанных на интегрируемом потенциале уравнения Шредингера.

\section{3. ГЕНЕРИРОВАНИЕ ТОЧНЫХ РЕШЕНИЙ ПРИ ЗАДАННОМ $U_{n}(t)$}

В качестве модельных “потенциалов" $U_{n}(t)$ рассмотрим следующие:

$$
\begin{gathered}
U_{n}(t)=\mu^{2} t^{2}, \\
U_{n}(t)=-\frac{2 \lambda_{0}}{\operatorname{ch}^{2}\left(\lambda_{0} t\right)} .
\end{gathered}
$$

Исследуем решения для потенциалов $(A),(B)$ с позиции возможности существования инфляционных режимов и выхода из инфляции.

Потенциал $(A)$. В этом случае решением уравнения (9) с нулевыми граничными условиями при $t \rightarrow \pm \infty$ являются решения вида

$$
\psi_{n}=A^{n} H_{s}(\mu t) e^{-\mu t^{2} / 2},
$$

где $A$ - постоянная, $H_{s}(\mu t)$ - полиномы Эрмита порядка $s$. Соответствующая этому решению эволюция масштабного фактора такова:

$$
a(t)=A H_{s}^{1 / n}(\mu t) e^{-\mu t^{2} / 2 n} .
$$

В простейшем случае $\psi_{n}=e^{-\mu t^{2} / 2}$. В этом случае зависимость от $n$ может быть исключена простым переопределением параметра $\mu$. Это решение рассматривалось в работе [1], поэтому мы не будем останавливаться на нем.

Выбор в качестве решения задачи (9) именно волновой функции основного состояния не является обязательным. Можно рассмотреть лежащие в $L^{2}$ решения, отвечающие возбужденным уровням, однако эти решения нельзя использовать на всем интервале, на котором они определены. Дело в том, что в соответствии с осцилляционной теоремой волновая функция $s$-го возбужденного уровня имеет $s$ нулей, каждый из которых в контексте космологических приложений означает наличие сингулярности, причем если $n>0$, то масштабный фактор будет обращаться в нуль, а если $n<0$, то масштабный фактор будет обращаться в бесконечность 
(сингулярность большого разрыва). Можно использовать решения для возбужденных уровней с номерами $s>1$ и рассматривать динамику, описываемую частью волновой функции на определенном интервале. Такая вселенная будет начинать и/или заканчивать свое существование в соответствующей сингулярности. Например, если в качестве решения (9) с “потенциалом" $U_{n}=\mu^{2} t^{2}$ взять функцию вида $\psi_{n} \sim H_{1}(\mu t) e^{-\mu t^{2} / 2}$, а $n$ считать равным -1 , то эволюцию масштабного фактора в такой вселенной можно записать в виде

$$
a(t)=\frac{a_{0} t_{0}}{t} e^{\mu\left(t^{2}-t_{0}^{2}\right) / 2} .
$$

Это решение описывает вселенную, масштабный фактор которой в момент времени $t=0$ равен бесконечности (сингулярность большого разрыва), при $t=\mu^{-1 / 2}$ масштабный фактор достигает минимального значения, а затем начинается инфляционная стадия, которая продолжается бесконечно долго. Используя формулы (10), (11), можно найти также асимптотику скалярного поля и потенциала при $t \sim 0$ и $t \rightarrow \infty$. Кинетический член плотности энергии скалярного поля отрицателен, т.е. эволюция (20) соответствует фантомному полю (о фантомных полях см., например, [12]-[16]). Решению (20) соответствует отрицательная космологическая константа $\Lambda=-3 \mu$.

Потенциал $(B)$. Решение для потенциала $(B)$ при $\lambda_{n}=-\lambda^{2} \leqslant 0$ (что соответствует неотрицательному значению космологической константы) имеет вид

$$
\psi_{n}=c_{1}\left(\lambda-\lambda_{0} \operatorname{th}\left(\lambda_{0} t\right)\right) e^{\lambda t}+c_{2}\left(\lambda+\lambda_{0} \operatorname{th}\left(\lambda_{0} t\right)\right) e^{-\lambda t}
$$

Если $\lambda=\lambda_{0}$, то формула (21) существенно упрощается:

$$
\psi_{n}=\frac{C}{\operatorname{ch}\left(\lambda_{0} t\right)} .
$$

Для положительных $n$ соответствующая функции $\psi_{n}$ эволюция масштабного фактора описывает инфляционный режим на интервале $\left(-\infty, t_{0}\right)$, где $t_{0}$ - точка перегиба функции $\operatorname{ch}^{1 / n}\left(\lambda_{0} t\right)$.

Интересный класс решений можно получить и в самом простом случае $\lambda_{n}=0$. Тогда (21) принимает вид:

$$
\psi_{n}=C \operatorname{th}\left(\lambda_{0} t\right) .
$$

Если $n \geqslant 1$, то решение для масштабного фактора будет описывать выход вселенной из сингулярного состояния в момент $t=0$ и ее асимптотический переход при $t \rightarrow \infty$ в стационарное состояние, причем $\ddot{a}<0$ в течение всей эволюции. Если же $0<n<1$, то появляется режим инфляции, продолжающийся до некоторого момента времени, а затем вселенная асимптотически переходит в стационарное состояние.

В случае $\lambda>\lambda_{0}, c_{1}>0, c_{2}=0$ имеется решение

$$
\begin{aligned}
\psi_{n} & =c_{1}\left(\lambda-\lambda_{0} \operatorname{th}\left(\lambda_{0} t\right)\right) e^{\lambda t}, \\
a & =c_{1}^{1 / n}\left(\lambda-\lambda_{0} \operatorname{th}\left(\lambda_{0} t\right)\right)^{1 / n} e^{\lambda t / n} .
\end{aligned}
$$


Это решение соответствует эволюции без сингулярностей и интересно тем, что при определенных значениях $\lambda_{0}, \lambda$ и $n$ формулы (23) описывают вселенную, которая испытывает стадию инфляционного расширения на некотором интервале $\left(0, t_{1}\right)$, затем происходит выход из инфляции, а потом, начиная с некоторого времени $t_{2}>t_{1}$, вселенная вновь расширяется в режиме инфляции. Для того чтобы определить, при каких условиях мы имеем такую картину, рассмотрим $\ddot{a}$ в нулевой момент времени. Используя (23), видим, что

$$
\ddot{a}(0) \sim(1-n) y^{4}-2 y^{2}+1,
$$

где введено обозначение $y=\lambda_{0} / \lambda<1$. Таким образом, вторая производная масштабного фактора в нулевой момент времени будет неотрицательной, если

$$
0<y^{2} \leqslant y_{0}^{2}=\frac{1-\sqrt{n}}{1-n}
$$

Дальнейшее исследование показывает, что если $n \geqslant 1$, то выполнение данного условия означает, что эволюция вселенной сразу выходит на режим инфляции. Если $y^{2}>y_{0}^{2}$, то решение (23) описывает эволюцию с появлением режима инфляции в некоторый момент времени $t_{0}>0$. При $n<1$ в узком интервале значений $y_{0}^{2}-\Delta<y^{2}<y_{0}^{2}$, где $\Delta \ll y_{0}^{2}$, имеется область неинфляционного расширения. Затем вселенная вновь выходит на режим ускоренного расширения. Например, если $n=0.25, \lambda_{0}=0.8 \lambda$, то вселенная ускоренно расширяется в интервале $(0,0.08 / \lambda)$, затем следует стадия неинфляционного расширения, длительность которой составляет приблизительно $0.4 / \lambda$. При $t>5 / \lambda$ масштабный фактор можно считать меняющимся по экспоненциальному закону $a \sim e^{4 \lambda t}$.

В заключение рассмотрим асимптотику потенциала скалярного поля на ранней стадии развития такой вселенной. В разложении ограничимся слагаемыми с точностью до первого порядка по времени. Используя (10), (11), имеем при $t \ll 1 / \lambda$

$$
\begin{aligned}
\phi(t) & \approx \phi_{0} \pm \sqrt{\frac{2}{3 n}} y^{2} \lambda t, \\
U(t) & \approx \frac{\lambda^{2}}{3}\left(\frac{3-n}{n^{2}} y^{4}-\frac{6}{n^{2}} y^{2}-\frac{2(3-n)}{n^{2}} y^{4}\left(1-y^{2}\right) \lambda t\right) .
\end{aligned}
$$

Таким образом, если есть первичная инфляции, то она соответствует режиму медленного скатывания. Потенциал медленно убывает с ростом/убыванием скалярного поля по линейному закону.

\section{4. ЗАКЛЮЧЕНИЕ}

В работе предложен относительно простой метод генерации точных решений уравнений Эйнштейна-Фридмана. Путем математических преобразований задача сведена к решению уравнения Шредингера для функции $a^{n}$ с “потенциалом", пропорциональным $n^{2} \rho-3 n\left(\rho+p / c^{2}\right) / 2$. Основное внимание уделено тем аспектам, которые связаны с изучением режима инфляции и выхода из него.

Трехпараметрические семейства решений демонстрируют гораздо более богатый репертуар поведения, чем изученные в работе [1] двухпараметрические решения. 
Тем не менее эти решения, как и решения, описанные в работе [1], показывают наличие инфляционных режимов при весьма общих предположениях. Это служит дополнительным указанием на то, что инфляция не является чем-то экзотическим и реализуемым лишь на весьма ограниченном классе моделей. Напротив, режим инфляции можно считать достаточно обычным явлением в космологии, не требующим для своего существования особых начальных условий.

В отличие от двухпараметрических решений, трехпараметрические решения могут демонстрировать несколько последовательных фаз инфляции, т.е. способны описывать не только режим инфляции, но и выход из этого режима без специальной настройки (подгонки) параметров. Более того, наличие таких решений можно рассматривать как косвенное указание на возможность существования единой, реалистичной модели, содержащей не только инфляционный режим (с выходом без тонкой настройки) на стадии ранней вселенной, но и более позднюю инфляцию, которую наша Вселенная, возможно, переживает в настоящее время.

Наконец, процедура построения трехпараметрических решений может быть повторена произвольное число $N$ раз, что приводит к $3 N$-параметрическим решениям. Наличие достаточно большого числа свободных параметров может рассматриваться как недостаток теории, а может, наоборот, служить удобным способом согласования теоретической модели с данными наблюдений. Мы рассчитываем вернуться к этому вопросу в дальнейших исследованиях.

Таким образом, описанный метод может оказаться весьма плодотворным в космологии.

Благодарности. Авторы выражают благодарность С. В. Червону за полезное обсуждение полученных результатов. Авторы также благодарны рецензенту за ценные замечания по основному тексту статьи. Работа выполнена при частичной поддержке РФФИ (грант № 08-02-91307-ИНД_а).

\section{Список литературы}

[1] В. М. Журавлев, С. В. Червон, В. К. Щиголев, ЖЭЭФ, 114:2 (1998), 406-417.

[2] J. D. Barrow, Phys. Rev. D, 49:6 (1994), 3055-3058.

[3] R. Maartens, D. R. Taylor, N. Roussos, Phys. Rev. D, 52:6 (1995), 3358-3364.

[4] G.F. R. Ellis, M. S. Madsen, Class. Quantum Grav., 8:4 (1991), 667-676.

[5] J.E. Lidsey, Class. Quantum Grav., 8:5 (1991), 923-933.

[6] J. D. Barrow, P. Saich, Class. Quantum Grav., 10:2 (1993), 279-283.

[7] P. Parson, J. D. Barrow, Class. Quantum Grav., 12:7 (1995), 1715-1721.

[8] A.V. Yurov, Phantom scalar fields result in inflation rather than Big Rip, arXiv: astro-ph/0305019.

[9] С. Д. Верещагин, А. В. Юров, ТМФ, 139:3 (2004), 405-422.

[10] A. V. Yurov, V. A. Yurov, Phys. Rev. D, 72:2 (2005), 026003.

[11] A. V. Yurov, A. V. Astashenok, V. A. Yurov, Gravit. Cosmol., 14:1 (2008), 8-16.

[12] J. Cepa, J. Astron. Astrophys., 422:3 (2004), 831-839.

[13] A. A. Andrianov, F. Cannata, A. Y. Kamenshchik, Phys. Rev. D, 72 (2005), 043531.

[14] A. V. Yurov, P. M. Moruno P. F. González-Díaz, Nucl. Phys. B, 759:1-2 (2006), 320-341.

[15] E. Elizalde, S. Nojiri, S. Odintsov, Phys. Rev. D, 70:4 (2004), 043539.

[16] S. Nojiri, S. Odintsov, Phys. Lett. B, 562 (2003), 147-152.

Поступила в редакцию 6.01.2008, после доработки 13.03.2008 